\title{
Super Chern-Simons Theory and
}

\section{Flat Super Connections on a Torus}

\author{
A. Miković and R. Picken \\ Departamento de Matemática and Centro de Matemática Aplicada \\ Instituto Superior Tecnico \\ Av. Rovisco Pais, 1049-001 Lisboa, Portugal \\ amikovic@math.ist.utl.pt, rpicken@math.ist.utl.pt
}

\begin{abstract}
We study the moduli space of a super Chern-Simons theory on a manifold with the topology $\mathbf{R} \times \Sigma$, where $\Sigma$ is a compact surface. The moduli space is that of flat super connections modulo gauge transformations on $\Sigma$, and we study in detail the case when $\Sigma$ is a torus and the supergroup is $O S p(m \mid 2 n)$. The bosonic moduli space is determined by the flat connections for the maximal bosonic subgroup $O(m) \times S p(2 n)$, while the fermionic moduli appear only for special parts of the bosonic moduli space, which are determined by a vanishing determinant of a matrix associated to the bosonic part of the holonomy. If the CS supergroup is the exponential of a super Lie algebra, the fermionic moduli appear only for the bosonic holonomies whose generators have zero determinant in the fermion-fermion block of the super-adjoint representation. A natural symplectic structure on the moduli space is induced by the super Chern-Simons theory and it is determined by the Poisson bracket algebra of the holonomies. We show that the symplectic structure of homogeneous connections is useful for understanding the properties of the moduli space and the holonomy algebra, and we illustrate this for the example of the $O S p(1 \mid 2)$ supergroup.
\end{abstract}

e-print archive: http://xxx.lanl.gov/math-ph/0008006 


\section{Introduction}

The study of Chern-Simons (CS) theories based on non-compact groups is important for understanding classical and quantum properties of threedimensional (3d) gravity theories [1]. One can show that pure $3 \mathrm{~d}$ general relativity is $I S O(1,2)$ (3d Poincare group) CS theory, while $3 \mathrm{~d}$ general relativity with a cosmological constant $\Lambda$ is a CS theory based on the group $S O(2,2)$ for $\Lambda<0$ (Anti-de-Sitter gravity), while for $\Lambda>0$ it is the $S O(1,3) \mathrm{CS}$ theory (de-Sitter gravity) [2,3]. Furthermore, $3 \mathrm{~d}$ supergravity is a super CS theory for the 3d super-Poincare group [2], AdS supergravity is a super CS for a certain class of supergroups $[4,6]$, one of which is $O S p(m \mid 2) \times O S p(n \mid 2)$ [3], while for de-Sitter supergravity the relevant group is $O S p(1 \mid 2 ; \mathbf{C})[5]$.

The moduli space of a CS theory represents the physical degrees of freedom (dof) and it is important for understanding the classical solutions and for the quantization. When the three-manifold has topology $\mathbf{R} \times \Sigma$, where $\Sigma$ is compact, then the moduli space for compact groups is well understood [7]. However, when the CS group is non-compact, there are few results. The 3d Poincare group case has been studied in [8] when $\Sigma$ is a torus $T^{2}$, and the moduli space is finite dimensional with a non-Hausdorf topology. The super-Poincare case for a torus has been studied in [9], and the novelty is that the fermionic moduli only appear for special parts of the bosonic moduli space. Also the structure of the $3 \mathrm{~d}$ super-Poincare algebra is such that there are no quadratic fermion contributions to the bosonic curvature two-form, so that the problem of constructing the moduli space is simpler than in the case of other relevant super groups.

Introducing a symplectic structure on the moduli space is important for understanding solutions and quantization. In the case of groups relevant for $3 \mathrm{~d}$ gravity, one can use the approach of [10], where traces of holonomies are used as basic variables. This was extended to the super de-Sitter case in [11]. However, the problem with using traces of holonomies is that it is difficult to understand the fermionic moduli space, basically because the trace is an even element of the Grassman algebra while fermionic moduli belong to the odd part. Hence it is better to consider the holonomy matrix elements, and their algebra in order to find fermionic observables. Even in 
the purely bosonic case there are advantages in working with the holonomy matrix elements [12].

In order to explore theses issues we consider a super CS theory for the $O S p(m \mid 2 n)$ group on $R \times T^{2}$. In section 2 we give a definition of a super CS theory for an arbitrary super Lie group and a three manifold $M$. In section 3 we specialize to the case $M=R \times \Sigma$ and describe the canonical formalism. In section 4 we introduce the holonomies for a super Lie group and describe how to determine the moduli space for $\Sigma=T^{2}$. In section 5 we study the moduli space for the $O S p(m \mid 2 n)$ supergroup and we determine the condition for the existence of the fermionic moduli. In section 6 we discuss the basic features of the symplectic structure on the moduli space, and show how the homogeneous connections can be used to extract information about the moduli space in the exponential sector of the moduli space. We extend these results to the case when the CS supergroup is the exponential of an arbitrary super Lie algebra. In section 7 we work out the $O S p(1 \mid 2)$ case in detail and we present our conclusions in section 8 .

\section{Super Chern-Simons Theory}

Let $g_{s}$ be a super Lie algebra, with a basis $T_{I}=\left\{J_{a}, Q_{\alpha}\right\}$, where $J_{a}$ are even elements and $Q_{\alpha}$ are odd elements. $g_{s}$ has a super Lie bracket [, \}, which satisfies

$$
[X, Y\}=(-1)^{|X||Y|+1}[Y, X\},
$$

as well as the super Jacobi identity

$$
[X,[Y, Z\}\}=[[X, Y\}, Z\}+(-1)^{|X||Y|}[Y,[X, Z\}\},
$$

where $X, Y$ and $Z$ are elements of definite parity $(|X|=0,1$ for an even, odd element respectively). The super Lie algebra $g_{s}$ is determined by the super Lie brackets of the basis elements

$$
\left[T_{I}, T_{J}\right\}=f_{I J}{ }^{K} T_{K},
$$

where $f_{I J}{ }^{K}$ are the structure constants, belonging to $\mathbf{R}$ or $\mathbf{C}$. 
The algebra $g_{s}$ can be represented by matrices so that the relations (3) take the following form

$$
\begin{aligned}
{\left[J_{a}, J_{b}\right] } & =f_{a b}{ }^{c} J_{c}, \\
{\left[J_{a}, Q_{\alpha}\right] } & =f_{a \alpha}{ }^{\beta} Q_{\beta}, \\
\left\{Q_{\alpha}, Q_{\beta}\right\} & =f_{\alpha \beta}{ }^{a} J_{a},
\end{aligned}
$$

where $[X, Y]=X Y-Y X$ and $\{X, Y\}=X Y+Y X$.

Let $B_{N}$ be a Grassmann algebra generated by $\left\{\theta_{1}, \ldots, \theta_{N}\right\}$, which satisfy

$$
\left\{\theta_{i}, \theta_{j}\right\}=0 \quad 1 \leq i, j \leq N .
$$

The natural basis of $B_{N}$ can be split into an even and odd part

$$
\begin{aligned}
E_{N} & =\left\{1, \theta_{i} \theta_{j}, \theta_{i} \theta_{j} \theta_{k} \theta_{l}, \ldots\right\} \\
O_{N} & =\left\{\theta_{i}, \theta_{i} \theta_{j} \theta_{k}, \ldots\right\},
\end{aligned}
$$

so that $B_{N}=L\left(E_{N}\right) \oplus L\left(O_{N}\right)=B_{N}^{+} \oplus B_{N}^{-}$. We can now define a Grassmann enveloping algebra of $g_{s}, B\left(g_{s}\right)$, as

$$
B\left(g_{s}\right)=\left\{X=X^{a} J_{a}+\chi^{\alpha} Q_{\alpha} \mid X^{a} \in B_{N}^{+}, \chi^{\alpha} \in B_{N}^{-}\right\} .
$$

Consider now a one-form $A$ on a three-manifold $M$, taking values in $B\left(g_{s}\right)$. If $\left\{x^{\mu}\right\}$ are coordinates on $M$, then

$$
A=A_{\mu}^{a}(x) d x^{\mu} J_{a}+\psi_{\mu}^{\alpha}(x) d x^{\mu} Q_{\alpha}=A_{\mu}^{I}(x) d x^{\mu} T_{I} .
$$

Note that in physics there is a restriction $A_{\mu}^{a} \in B_{0}=\mathbf{R}$ or $\mathbf{C}$ while $\psi_{\mu}^{\alpha} \in$ $B_{1}=L\left(\theta_{i}\right)$. The super Chern-Simons theory associated to a super Lie group $G_{s}$, whose Lie algebra is $B\left(g_{s}\right)$, is defined by the action functional

$$
S[A]=\int_{M} \operatorname{str}\left(A d A+\frac{2}{3} A \wedge A \wedge A\right),
$$

where $d$ is the exterior derivative, and str is the super trace (invariant bilinear form on $g_{s}$ ), given by

$$
\operatorname{str}\left(J_{a} J_{b}\right)=\eta_{a b}=\eta_{b a} \quad, \quad \operatorname{str}\left(Q_{\alpha} Q_{\beta}\right)=C_{\alpha \beta}=-C_{\beta \alpha} \quad \operatorname{str}\left(J_{a} Q_{\alpha}\right)=0 .
$$


This is denoted as

$$
\operatorname{str}\left(T_{I} T_{J}\right)=\eta_{I J}
$$

The equations of motion are the extremal points of $S[A]$

$$
\frac{\delta S}{\delta A}=d A+A \wedge A=F=0
$$

which are satisfied by the flat $G_{s}$ connections on $M$. This can be written in a more explicit form

$$
\begin{aligned}
& F^{a}=d A^{a}+f_{b c}^{a} A^{b} \wedge A^{c}+f_{\alpha \beta}{ }^{a} \psi^{\alpha} \wedge \psi^{\beta}=0 \\
& F^{\alpha}=d \psi^{\alpha}+f_{a \beta}{ }^{\alpha} A^{a} \wedge \psi^{\beta}=0
\end{aligned}
$$

The super CS theory is invariant under the gauge transformations $g$ : $M \rightarrow G_{s}$, so that the connection $A$ and the connection

$$
\tilde{A}=g A g^{-1}+g d g^{-1}
$$

are equivalent. Hence the moduli space is the space of flat connections modulo gauge transformations.

\section{$3 \quad M=\mathbf{R} \times \Sigma$ case}

We study now the case when $M=\mathbf{R} \times \Sigma$. In this case there is a natural split of the coordinates $x^{\mu}=\left\{t, x^{i}\right\}$ where $t \in \mathbf{R}$ and $x^{i}$ are coordinates on $\Sigma$. This induces the split for the one forms $A_{\mu}=\left\{A_{0}, A_{i}\right\}$, so that the action can be written in the Hamiltonian form

$$
S=\int_{t_{1}}^{t_{2}} d t \int_{\Sigma} d^{2} x\left[E_{I}^{i} \dot{A}_{i}^{I}-A_{0}^{I} G_{I}-\lambda_{i}^{I} \theta_{I}^{i}\right]
$$

where $G_{I}$ are the first-class Gauss constraints

$$
G_{I}=\partial_{i} E_{I}^{i}+f_{I K}^{J} A_{i}^{K} E_{J}^{i}=0
$$


while $\theta_{I}^{i}$ are the second-clsss constraints

$$
\theta_{I}^{i}=E_{I}^{i}-\eta_{I J} \epsilon^{i j} A_{j}^{J}=0 .
$$

Here $E_{I}^{i}$ is the canonically conjugate momentum to $A_{i}^{I}$ and $\epsilon_{i j}$ is the antisymmetric tensor density on $\Sigma$. One can introduce the super Poisson brackets $(\mathrm{PB})$ for the functions on the phase space $(E, A)[16]$

$$
\{F(x), G(y)\}_{P B}=\int_{\Sigma} d^{2} z\left(\frac{\delta F(x)}{\delta E_{I}^{i}(z)} \frac{\delta G(y)}{\delta A_{i}^{I}(z)}-(-1)^{\left|A_{i}^{I} \| E_{I}^{i}\right|} \frac{\delta F(x)}{\delta A_{i}^{I}(z)} \frac{\delta G(y)}{\delta E_{I}^{i}(z)}\right),
$$

so that

$$
\left\{E_{I}^{i}(x), A_{j}^{J}(y)\right\}_{P B}=\delta_{j}^{i} \delta_{I}^{J} \delta(x-y)
$$

The first-class constraints form a closed algebra under the Poisson brackets

$$
\left\{G_{I}(x), G_{J}(y)\right\}_{P B}=f_{I J}{ }^{K} \delta(x-y) G_{K}(y),
$$

and $Q=\int_{\Sigma} d^{2} x \epsilon^{I}(x) G_{I}(x)$ generate the infinitesimal (identity connected) gauge transformations

$\delta A(x)=\{Q, A(x)\}_{P B}, \quad \delta E(x)=\{Q, E(x)\}_{P B}, \quad \delta A_{0}^{I}=\dot{\epsilon}^{I}+f_{J K}{ }^{I} A_{0}^{J} \epsilon^{K}$.

Hence the moduli space is the constrained phase space modulo the gauge transformations. Because of the second class constraint, the Gauss constraint becomes

$$
\epsilon^{i j} F_{i j, I}=0
$$

where $F=d A+A \wedge A$. Therefore the moduli space $\mathcal{M}$ is that of flat $G_{s}$ connections on $\Sigma$.

The PB algebra of the second-class constraints does not close on the constrained phase space, and hence the PB (21) will not induce a good PB on $\mathcal{M}$. This can be remedied by introducing a second $\mathrm{PB}$, the Dirac bracket

$$
\{F, G\}^{*}=\{F, G\}_{P B}-\left\{F, \theta_{\alpha}\right\}_{P B} \Delta^{\alpha \beta}\left\{\theta_{\beta}, G\right\}_{P B},
$$


where

$$
\Delta^{\alpha \beta}\left\{\theta_{\beta}, \theta_{\gamma}\right\}_{P B}=\delta_{\gamma}^{\alpha},
$$

and $\alpha, \beta, \gamma$ denote discrete and continuous indices. The DB is well defined on the constraint surface, since $\left\{\theta_{1}, \theta_{2}\right\}^{*}=0$. The basic Dirac brackets are

$$
\left\{A_{i}^{I}(x), A_{j}^{J}(y)\right\}^{*}=\epsilon_{i j} \eta^{I J} \delta(x-y)
$$

which will induce a symplectic structure on $\mathcal{M}$. The DB (28) induces a symplectic 2 -form

$$
\Omega=\int_{\Sigma} \operatorname{str}\left(\delta_{1} A \wedge \delta_{2} A\right)
$$

which is the supergroup generalization of the Atiyah-Bott symplectic form [14].

\section{Holonomies and the moduli space}

The standard approach for determining the moduli of flat connections is to use the holonomies. We will use the same approach for the case of super Lie groups, so let $U$ be a homomorphism from the fundamental group of $\Sigma$, $\pi_{1}(\Sigma)$, to $G_{s}$ defined by

$$
U(\gamma)=U(s=2 \pi) \quad, \quad \frac{d U(s)}{d s}=A_{\mu}(s) \frac{d x^{\mu}}{d s} U(s)
$$

where $x^{\mu}(s):[0,2 \pi] \rightarrow M$ parametrizes the loop $\gamma$, and $U(0)=I d$. Since we use a matrix representation for $g_{s}$, which are $(m+n) \times(m+n)$ matrices, the corresponding $G_{s}$ will be given by $(m+n) \times(m+n)$ super matrices

$$
g=\left(\begin{array}{cc}
A & B \\
C & D
\end{array}\right)
$$

where $A, D$ are $m \times m$ and $n \times n$ matrices respectively, with entries in $B_{N}^{+}$, while $B$ and $C$ are $m \times n$ and $n \times m$ matrices respectively, with entries in $B_{N}^{-}$. Given a $B\left(g_{s}\right)$ one can always obtain a super Lie group by the exponential 
map $g=e^{X}$, but as in the case of ordinary Lie groups, one can have $G_{s}$ with elements $g \neq e^{X}$.

The $\pi_{1}(\Sigma)$ for a Riemann surface $\Sigma$ is generated by the canonical cycles of $\Sigma,\left(a_{i}, b_{i}\right), i=1, \ldots, g$, where $g$ is the genus of $\Sigma$. The generators satisfy a constraint

$$
\prod_{i=1}^{g} a_{i}^{-1} b_{i}^{-1} a_{i} b_{i}=1 .
$$

In the case of the torus the constraint becomes $a_{1} b_{1}=b_{1} a_{1}$, so that the corresponding holonomies satisfy

$$
U_{1} U_{2}=U_{2} U_{1}
$$

Since a holonomy transforms by conjugation under gauge transformations, the moduli space will be determined by commuting pairs $\left(U_{1}, U_{2}\right)$ from $G_{s}$ modulo conjugation, i.e.

$$
\left(U_{1}, U_{2}\right) \sim\left(\mathcal{S} U_{1} \mathcal{S}^{-1}, \mathcal{S} U_{2} \mathcal{S}^{-1}\right) \quad, \quad \mathcal{S} \in G_{s}
$$

\section{$5 \quad O S p(m \mid 2 n)$ supergroup}

We now concentrate on the $O S p(m \mid 2 n)$ Lie group case. It can be defined as a group of super matrices $M$

$$
M^{s t} H M=H=\operatorname{diag}\left(I_{m}, C_{2 n}\right)
$$

where $C^{T}=-C$ and $C^{2}=-I_{2 n}$. If we label $M$ as

$$
M=\left(\begin{array}{cc}
a & \xi \\
\chi & A
\end{array}\right)
$$

and use

$$
M^{s t}=\left(\begin{array}{cc}
a^{T} & \chi^{T} \\
-\xi^{T} & A^{T}
\end{array}\right)
$$


then the relations (35) imply

$$
\begin{aligned}
a^{T} a+\chi^{T} C \chi & =I_{m} \\
a^{T} \xi+\chi^{T} C A & =0 \\
-\xi^{T} \xi+A^{T} C A & =C .
\end{aligned}
$$

These relations can be solved by expanding the matrices $a, A, \chi, \xi$ in the Grassman algebra $B_{N}$ basis as

$$
X=X_{0}+X_{1} \theta^{1}+X_{2} \theta^{2}+\ldots+X_{N} \theta^{N},
$$

where $\theta^{k}=\theta_{i_{1}} \cdots \theta_{i_{k}}$ and we have suppressed the $i_{l}$ indices on $X_{k}$. Note that for physics applications $N \geq 2 m n$, otherwise $N$ is an arbitrary natural number.

The relations (38) and (40) imply

$$
a_{0}^{T} a_{0}=I_{m} \quad, \quad A_{0}^{T} C A_{0}=C,
$$

so that $a_{0} \in O(m)$, while $A_{0} \in S p(2 n)$. This implies that $a$ and $A$ are always invertible, so that the relation (39) gives

$$
\xi=-\left(a^{T}\right)^{-1} \chi^{T} C A,
$$

which means that there is only one independent fermionic matrix. Hence if $\chi=0$, then $\xi=0$ and therefore $a \in O(m)$ while $A \in S p(2 n)$, so that one obtains the maximal bosonic subgroup $O(m) \times S p(2 n)$.

If $M$ is a holonomy matrix $U$, we would like to know how the fermion $\chi$ transforms under the gauge transformation

$$
\tilde{U}=\mathcal{S} U \mathcal{S}^{-1}=\left(\begin{array}{cc}
\tilde{a} & \tilde{\xi} \\
\tilde{\chi} & \tilde{A}
\end{array}\right)
$$

where $\mathcal{S} \in O S p(m \mid 2 n)$. If

$$
\mathcal{S}=\left(\begin{array}{ll}
s & \delta \\
\sigma & S
\end{array}\right)
$$


then

$$
\mathcal{S}^{-1}=\left(\begin{array}{cc}
\bar{s}^{-1} & -s^{-1} \delta \bar{S}^{-1} \\
-S^{-1} \sigma \bar{s}^{-1} & \bar{S}^{-1}
\end{array}\right)
$$

where $\bar{s}=s-\delta S^{-1} \sigma$ and $\bar{S}=S-\sigma s^{-1} \delta$. By using (46), the relation (44) gives

$$
\tilde{\chi}=\left(\sigma a+S \chi-\sigma \xi S^{-1} \sigma-S A S^{-1} \sigma\right) \bar{s}^{-1}
$$

Therefore if we want $\tilde{\chi}=0$, then (47) implies

$$
\sigma a+S \chi-\sigma \xi S^{-1} \sigma-S A S^{-1} \sigma=0
$$

This can be considered as an equation for $\sigma$, which can be written in components of a $B_{N}$ basis as

$$
\sigma_{2 k+1} a_{0}-S_{0} A_{0} S_{0}^{-1} \sigma_{2 k+1}=-S_{0} \chi_{2 k+1}+\zeta_{2 k+1}\left(\sigma_{1}, \ldots, \sigma_{2 k-1}\right)
$$

where $\zeta_{1}=0$. Therefore (49) can be solved iteratively for $\sigma$ as a function of $a, A, \chi$ and $s, S$ provided that the linear operator $\hat{A}$ determined by

$$
\hat{A} \sigma=\sigma a_{0}-S_{0} A_{0} S_{0}^{-1} \sigma
$$

is invertible. It can be written as a $2 m n \times 2 m n$ matrix

$$
\hat{A}_{0}=a_{0}^{T} \otimes I_{2 n}-I_{m} \otimes S_{0} A_{0} S_{0}^{-1}
$$

so that invertability is equivalent to $\operatorname{det} \hat{A}_{0} \neq 0$. Note that

$$
\hat{S}^{-1} \hat{A}_{0} \hat{S}=a_{0}^{T} \otimes I_{2 n}-I_{m} \otimes A_{0}
$$

where $\hat{S}=I_{m} \otimes S_{0}$, so that

$$
\operatorname{det}\left(a_{0}^{T} \otimes I_{2 n}-I_{m} \otimes S_{0} A_{0} S_{0}^{-1}\right)=\operatorname{det}\left(a_{0}^{T} \otimes I_{2 n}-I_{m} \otimes A_{0}\right)
$$

In the case when $\operatorname{det} \hat{A}_{0} \neq 0$ one can determine all the components of $\sigma$ and hence set all of the components of $\tilde{\chi}$ to zero. This then means that the holonomy matrix $U$ is equivalent to a holonomy matrix of the $O(m) \times S p(2 n)$ 
subgroup. If $U_{1}$ is such a matrix, i.e. $U_{1}=\operatorname{diag}(a, A)$ then $U_{2}$ must be also, since the commutativity implies

$$
a b=b a \quad, \quad A B=B A \quad, \quad \mu a=A \mu \quad, \quad a \nu=\nu A,
$$

where

$$
U_{2}=\left(\begin{array}{cc}
b & \nu \\
\mu & B
\end{array}\right)
$$

Since $\operatorname{det}\left(a^{T} \otimes I_{2 n}-I_{m} \otimes A\right) \neq 0$ then $\mu a-A \mu=0$ implies $\mu=0$, and hence $U_{2}=\operatorname{diag}(b, B)$. Hence the moduli space is that of the $O(m) \times S p(2 n)$ Lie group, and there are no fermionic moduli.

Note that this bosonic moduli space will be larger then the moduli space of non-super CS based on the $O(m) \times S p(2 n)$ Lie group because the holonomy matrices in the former case belong to the $B_{N}^{+}$Grassman algebra while in the latter case the holonmy matrices are real numbers. We can avoid these extra bosonic degrees of freedom by restricting the even part of the CS connection $A$ from $B_{N}^{+}$to real numbers.

The fermionic moduli will arise in the case when $\operatorname{det} \hat{A}_{0}=0$ and $\operatorname{det} \hat{B}_{0}=$ 0 where

$$
U_{1}=\left(\begin{array}{cc}
a & \xi \\
\chi & A
\end{array}\right) \quad, \quad U_{2}=\left(\begin{array}{cc}
b & \nu \\
\mu & B
\end{array}\right)
$$

The zero commutator implies

$$
\begin{gathered}
a b+\xi \mu=b a+\nu \chi \\
A B+\chi \nu=B A+\mu \xi \\
\chi b+A \mu=\mu a+B \chi \\
a \nu+\xi B=B \xi+\nu A .
\end{gathered}
$$

Let $\operatorname{rank}\left(\hat{A}_{0}\right)=r<2 m n$, so that $r$ components of $\chi$ can be set to zero. Although the non-zero components are not gauge invariant, they cannot 
be set to zero by a gauge transformation. We will show in the following sections how to define the gauge invariant fermion components, but for our present purposes it sufficient to know that the number of non-zero fermion components is gauge invariant. If $\operatorname{rank}\left(\hat{B}_{0}\right)=r^{\prime}$, then one can prove that $r=r^{\prime}$. From (57) and (58) it follows

$$
\left[a_{0}, b_{0}\right]=0 \quad, \quad\left[A_{0}, B_{0}\right]=0,
$$

while from (59) it follows

$$
A_{0} \mu_{1}-\mu_{1} a_{0}=B_{0} \chi_{1}-\chi_{1} b_{0}
$$

The relation (62) can be rewritten as

$$
\hat{A}_{0} \hat{\mu}_{1}=\hat{B}_{0} \hat{\chi}_{1}
$$

From (61) it follows that $\left[\hat{A}_{0}, \hat{B}_{0}\right]=0$ and hence these matrices can be simultaneosly diagonalised (or put in the block-diagonal form). If $r^{\prime}>r$, then from 63 it follows that $r^{\prime}$ components of $\chi$ vanish, which is in contradiction with the assumption that only $r$ components of $\chi$ vanish. Hence $r^{\prime} \leq r$. If $r^{\prime}<r$ then by reversing $\mu$ and $\chi$ and by the same argument it follows $r \leq r^{\prime}$ so that $r=r^{\prime}$. Hence the number of the independent fermionic (odd) moduli is equal to $2(2 n m-r)$.

The total number of real (complex) parameters for the fermionic phasespace is $(2 n m-r) e^{2 n m}$. Physics expectation is $(2 n m-r) 4 n m$, i.e. only $\chi_{1}$ and $\mu_{1}$. This happens when $\psi \in B_{1}$.

\section{Symplectic structure of the moduli space}

We now discuss some aspects of the symplectic structure on the moduli space which will be useful for an understanding of the results we have derived so far. A natural symplectic structure on $\mathcal{M}$ can be induced by the symplectic structure (29) on the space of connections. By using the definition of $U(\gamma)$ and (28) one obtains

$$
\begin{aligned}
\left\{U_{A}^{B}(\gamma), U_{C}^{D}(\sigma)\right\}^{*}= & 2 s(\gamma, \sigma) \sum_{E, F, G, H}(-1)^{[(|B|-|E|)(|A|-|E|)+(|C|-|H|)(|D|-|H|)]} \\
& U_{A}^{E}\left(\gamma_{i}\right)\left(T^{I}\right)_{E}^{F} U_{F}^{B}\left(\gamma_{f}\right) U_{C}^{G}\left(\sigma_{i}\right)\left(T_{I}\right)_{G}^{H} U_{H}^{D}\left(\sigma_{f}\right)
\end{aligned}
$$


for loops with at most one intersection, where $s(\gamma, \sigma)$ is the intersection number [11]. From this algebra one can find in principle the PB algebra of the coordinates on the moduli space and hence the symplectic form. However, this is beyond the scope of this paper, and instead we will analyse a simpler algebra, induced by the homogeneous connections, since it will give a very good idea of the moduli space, especially of the fermionic moduli.

Consider the holonomies which are the exponentials of the Lie super algebra, i.e. the subgroup $g_{s}=\exp X$. Then $U_{k}$ are given by

$$
U_{k}=\exp \left(2 \pi A_{k}^{a} J_{a}+2 \pi \psi_{k}^{\alpha} Q_{\alpha}\right)
$$

where $A_{k}$ and $\psi_{k}$ are constants on $\Sigma$. These correspond to the homogenous sector of the moduli space, for which the corresponding connections can be written as

$$
A=A_{1} d \theta+A_{2} d \phi=\left(A_{1}^{a} J_{a}+\psi_{1}^{\alpha} Q_{\alpha}\right) d \theta+\left(A_{2}^{a} J_{a}+\psi_{2}^{\alpha} Q_{\alpha}\right) d \phi
$$

where $\theta, \phi \in[0,2 \pi]$ parametrize the $a, b$ cycles of the torus. Hence $\left[U_{1}, U_{2}\right]=$ 0 is equivalent to $\left[A_{1}, A_{2}\right]=0$ which gives the constraints

$$
\begin{gathered}
G^{a}=f_{b c}{ }^{a} A_{1}^{b} A_{2}^{c}+f_{\alpha \beta}{ }^{a} \psi_{1}^{\alpha} \psi_{2}^{\beta}=0 \\
G^{\alpha}=f_{a \beta}{ }^{\alpha}\left(A_{1}^{a} \psi_{2}^{\beta}-A_{2}^{a} \psi_{1}^{\beta}\right)=0
\end{gathered}
$$

These are first-class constraints and form an $\operatorname{osp}(m \mid 2 n)$ algebra under the Poisson brackets (28), which for the homogeneous sector become

$$
\left\{A_{k}^{a}, A_{j}^{b}\right\}^{*}=\epsilon_{k j} \eta^{a b} \quad, \quad\left\{\psi_{k}^{\alpha}, \psi_{j}^{\beta}\right\}^{*}=\epsilon_{k j} C^{\alpha \beta} .
$$

The relations (69) imply that the space $A, \psi$ is the usual phase space, i.e. half of the parameters are coordinates and the other half are the momenta.

According to the theory of solving the constraints in phase space [15, $16,17]$, the constraints (67) and (68) can be solved in the following manner. In the bosonic sector, if we require that $A_{k}$ are ordinary numbers, then the bosonic constraint implies that $A_{k}^{a} J_{a}$ belong to the same abelian subgroup, and hence $A_{k}^{a}=\mathcal{A}_{k} c^{a}$. In the fermionic sector, let $r$ be the number of independent fermionic constraints $G^{\tilde{\alpha}}$. $r$ will be given by the rank of the 
matrix $c^{a} f_{a \alpha}{ }^{\beta}$, and hence one can impose $r$ fermionic gauge-fixing conditions $\chi^{\tilde{\alpha}}=0$ such that

$$
\operatorname{det}\left\|\left\{G^{\tilde{\alpha}}, \chi^{\tilde{\beta}}\right\}^{*}\right\| \neq 0
$$

This gives $4 n m-r-r=2(2 n m-r)$ independent fermionic phase-space coordinates, while in the bosonic sector there at most two ${ }^{*}$ independent phase space coordinates $\mathcal{A}_{1}$ and $\mathcal{A}_{2}$.

Therefore the condition for the existence of the fermionic moduli for the exponential sector becomes

$$
\operatorname{det}\left\|c^{a} f_{a \alpha}^{\beta}\right\|=0 .
$$

Note that our analysis for the exponential sector is general, and applies to any super Lie group. Hence one finds all inequivalent (with respect to conjugation) Abelian subgroups of the maximal bosonic subgroup, and checks the condition (71). There is also an additional constraint

$$
f_{\alpha \beta}{ }^{a} \psi_{1}^{\alpha} \psi_{2}^{\beta}=0
$$

coming from the bosonic constraint (67). The constraint (72) will serve to determine the gauge-fixing functions $\chi^{\tilde{\alpha}}=0$, i.e. $\chi^{\tilde{\alpha}}$ will be linear in $\psi_{k}$ such that (72) is satisfied as well as the condition (70) (see section 7).

Also note that the matrix $J_{\alpha}^{\beta}=c^{a} f_{a \alpha}{ }^{\beta}$ is the representation of the generator of the Abelian subgroup to which the bosonic holonomy belongs, and it is the fermion-fermion block in the super-adjoint representation of the super Lie algebra $\left(T_{I}\right)_{J}^{K}=f_{I J}{ }^{K}$. In the $\operatorname{osp}(m \mid 2 n)$ case the condition $\operatorname{det} \hat{A}_{0}=0$ applies to any holonomy, and in the exponential sector it is equivalent to (71).

\section{$7 \quad O S p(1 \mid 2)$ case}

We now present explicit constructions in the simplest non-trivial case of the $O S p(1 \mid 2)$ supergroup. In this case we have $m=n=1$, so that $a$ is a

*There may be a further constraint for some Abelian subgroups 
Grassman number and $A$ is an $S p(2)=S L(2)$ matrix, and we take a $B_{2}$ Grassman algebra. The $\operatorname{osp}(1 \mid 2)$ algebra can be represented by matrices satisfying

$$
\begin{aligned}
{\left[J_{a}, J_{b}\right] } & =\epsilon_{a b}^{c} J_{c}, \\
{\left[J_{a}, Q_{\alpha}\right] } & =\left(\sigma_{a}\right)_{\alpha}{ }^{\beta} Q_{\beta} \\
\left\{Q_{\alpha}, Q_{\beta}\right\} & =\left(\sigma^{a}\right)_{\alpha \beta} J_{a},
\end{aligned}
$$

where $a=0,1,2$ and the matrices $\left(\sigma_{a}\right)_{\alpha}{ }^{\beta}$ are

$$
\sigma_{0}=\left(\begin{array}{cc}
0 & 1 \\
-1 & 0
\end{array}\right) \quad, \quad \sigma_{1}=\left(\begin{array}{cc}
1 & 0 \\
0 & -1
\end{array}\right) \quad, \quad \sigma_{2}=\left(\begin{array}{cc}
0 & 1 \\
1 & 0
\end{array}\right) .
$$

The indices $a$ and $\alpha$ are raised and lowered by $\eta_{a b}=\operatorname{diag}(-1,1,1)$ and $C_{\alpha \beta}=\epsilon_{\alpha \beta}$. The relevant matrix representation of the generators of the osp $(1 \mid 2)$ algebra is given by

$$
J_{a}=\left(\begin{array}{cc}
1 & 0 \\
0 & \sigma_{a}
\end{array}\right) \quad, \quad Q_{\alpha}=\left(\begin{array}{cc}
0 & -c_{\alpha}^{T} C \\
c_{\alpha} & 0
\end{array}\right)
$$

where $c_{1}^{T}=(1,0)$ and $c_{2}^{T}=(0,1)$. The exponential map gives only a subgroup of the full group.

For the group we have $a_{0}= \pm 1$, and inequivalent bosonic Abelian subgroups are given by

$$
\left(\begin{array}{cc}
1 & 0 \\
0 & e^{\phi \sigma_{0}}
\end{array}\right),\left(\begin{array}{cc}
1 & 0 \\
0 & e^{v \sigma_{1}}
\end{array}\right),\left(\begin{array}{cc}
1 & 0 \\
0 & e^{c\left(\sigma_{0}+\sigma_{2}\right)}
\end{array}\right)
$$

where $\sigma_{0}, \sigma_{1}$ and $\sigma_{+}=\sigma_{0}+\sigma_{2}$ generate non-equvalent abelian subalgebras of $s l(2)$.

Since any element of $S L(2)$ can be written as $\pm e^{X}$, the bosonic conjugacy classes will be given by pairs

$$
\left(U_{1}, U_{2}\right)=\left(\left(\begin{array}{cc}
a_{0} & 0 \\
0 & \epsilon_{1} e^{X}
\end{array}\right),\left(\begin{array}{cc}
b_{0} & 0 \\
0 & \epsilon_{2} e^{Y}
\end{array}\right)\right)
$$


where $\epsilon_{k}= \pm 1$ and $X$ and $Y$ belong to the same Abelian subalgebra of $s l(2)$ which is not the $s o(2)$ subalgebra. When $X, Y \in s o(2)$, then

$$
\left(U_{1}, U_{2}\right)=\left(\left(\begin{array}{cc}
a_{0} & 0 \\
0 & e^{X}
\end{array}\right),\left(\begin{array}{cc}
b_{0} & 0 \\
0 & e^{Y}
\end{array}\right)\right) .
$$

Hence there will be $2 \cdot 16+4=36$ sectors of bosonic conjugacy classes.

The fermionic moduli will exist for holonomies which satisfy the condition $\operatorname{det} \hat{A}_{0}=0$, which becomes

$$
\operatorname{det}\left(a_{0} I_{2}-A_{0}\right)=0
$$

This is solved by

$$
a_{0}= \pm 1 \quad, \quad A_{0}=\left(\begin{array}{cc} 
\pm 1 & c \\
0 & \pm 1
\end{array}\right) .
$$

Note that $A_{0}= \pm e^{L}$, where $L=c \sigma_{+}$, i.e. $e^{L}$ belongs to the $G L(1)$ subgroup of $S L(2)$. Hence $\operatorname{rank} \hat{A}_{0}=1$, and therefore there will be $4-1-1=2$ fermionic moduli. The corresponding holonomy matrices are given by

$$
U_{k}=\left(\begin{array}{cc} 
\pm 1 & \xi_{k} \\
\chi_{k} & \pm e^{L_{k}}
\end{array}\right)
$$

where $\chi_{k}=\left(0, \mu_{k}\right)^{T}$ and $\xi_{k}=-a_{0} \chi_{k}^{T} C A_{0}$. Hence there are $2 \cdot 2=4$ different sectors of conjugacy classes $\left(U_{1}, U_{2}\right)$ which contain fermions. One can have more general solutions by replacing $A_{0}$ by $A_{0}+A_{2} \theta_{1} \theta_{2}$ where $A_{0}^{T} C A_{2}+$ $A_{2}^{T} C A_{0}=0$, but as we discussed, we only consider physical connections. Since $U_{k}$ belong to the parabolic conjugacy class of $S L(2)$, we will have $c_{1}^{2}+c_{2}^{2}=1[9]$.

For the sector where $U=\exp X$, we have

$$
U_{k}=\exp 2 \pi\left(\mathcal{A}_{k} \sigma_{+}+\psi_{k}^{\alpha} Q_{\alpha}\right)
$$

where $\sigma_{+}=\sigma_{0}+\sigma_{2}$ and

$$
\left\{\mathcal{A}_{1}, \mathcal{A}_{2}\right\}^{*}=1 \quad, \quad\left\{\psi_{1}^{\alpha}, \psi_{2}^{\beta}\right\}^{*}=C^{\alpha \beta}
$$


The fermionic constraint yields

$$
\mathcal{A}_{1} \psi_{2}^{+}-\mathcal{A}_{2} \psi_{1}^{+}=0
$$

where $\psi^{\alpha}=\left(\psi^{+} \psi^{-}\right)$. By inserting the fermionic constraint into the bosonic constraint, one obtains

$$
\mathcal{A}_{1} \psi_{2}^{-}-\mathcal{A}_{2} \psi_{1}^{-}=0 \text {. }
$$

This condition represents a consistent gauge-fixing choice, so that there exists a canonical transformation $\psi_{k} \rightarrow \tilde{\psi}_{k}$ such that

$$
\tilde{\psi}_{1}=(\Pi, \tilde{\Pi})^{T} \quad, \quad \tilde{\psi}_{2}=(\tilde{\psi},-\psi)^{T},
$$

and the constraints (86) and (87) become equivalent to $\tilde{\Pi}=0$ and $\tilde{\psi}=0$ [17]. With such a choice of fermionic variables, and the fact that $\mathcal{A}_{1}=p$ and $\mathcal{A}_{2}=q$ where $\{p, q\}^{*}=1$, one can now express $c_{k}$ and $\chi_{k}$ in terms of basic canonical variables, and work out their PB. Constructing the canonical transformation $\psi_{k} \rightarrow \tilde{\psi}_{k}$ may not be easy, but one can show that $U_{1}=\exp 2 \pi\left(p \sigma_{+}+\frac{p}{q} c^{\alpha}(p, q) Q_{\alpha} \psi\right) \quad, \quad U_{2}=\exp 2 \pi\left(q \sigma_{+}+c^{\alpha}(p, q) Q_{\alpha} \psi\right)$,

where $c^{\alpha}$ can be determined from the requirement that $\psi_{2}^{\alpha}=c^{\alpha}(p, q) \psi+\cdots$ where $\cdots$ represent terms which vanish on the constraint surface.

For the non-exponential sector one can use the holonomy matrix algebra, but in some cases it is possible to obtain explicit expressions in terms of the basic canonical variables. For example, in the sector $a_{0}=1, A_{0}=-e^{X}$ (which has no fermionic moduli) one can construct the matrices $U(\phi)$ and $U(\theta)$ as

$$
U(\phi)=\operatorname{diag}(1, R(\phi / 2)) \exp \left(\mathcal{A}_{1} \sigma \phi+\psi_{1}^{\alpha} Q_{\alpha} \phi\right),
$$

where $R(\phi / 2)$ is the rotation matrix for angle $\phi / 2$, and $U(\theta)$ is given by replacing $\phi$ with $\theta$ in $U(\phi)$ and $\mathcal{A}_{1}$ with $\mathcal{A}_{2}$, so that $U(0)=I d$ and $U(2 \pi)=$ $U_{1,2}$. Hence the corresponding flat connections are given by

$$
A_{1}=U^{-1}(\phi) \partial_{\phi} U(\phi) \quad, \quad A_{2}=U^{-1}(\theta) \partial_{\theta} U(\theta)
$$


and they are not constant on $T^{2}$, but they are expressed in terms of constant moduli with simple PB. The moduli $\mathcal{A}_{k}$ satisfy the same constraints as in the homogeneous case, and the same results apply for them. However, this trick does not work for the holonomies with $a_{0}=-1$ and $A_{0}=-e^{X}$.

Since $c_{1}^{2}+c_{2}^{2}=1$, the symplectic structure for the bosonic moduli becomes degenerate. In the exponential sector this is equivalent to $p^{2}+q^{2}=1$ so that $p=\cos t, q=\sin t$ which suggests a dynamical interpretation as a harmonic oscillator with energy $E=1 / 2$.

The degeneracy of the bosonic symplectic structure for holonomies with fermions appears because the group is small. For bigger groups, there is generically no such degeneracy. For example, for $O S p(2 \mid 2)$ case we have $a_{0}$ is an $O(2)$ matrix while $A_{0}$ is an $S L(2)$ matrix. In the sector where $a_{0} \in S O(2)$, so that $a_{0}=R(\phi)$, the condition for the existence of the fermionic moduli is

$$
\operatorname{det} \hat{A}_{0}=\operatorname{det}\left(a_{0}^{T} \otimes I_{2}-I_{2} \otimes A_{0}\right)=\left(2 \cos \phi-\operatorname{tr} A_{0}\right)^{2}=0,
$$

Hence $A_{0}$ belongs to the $S O(2)$ subgroup of the $S L(2)$, and therefore the bosonic conjugacy classes which have the fermionic moduli are

$$
U_{1}=\operatorname{diag}\left(R\left(\phi_{1}\right), R\left( \pm \phi_{1}\right)\right), U_{2}=\operatorname{diag}\left(R\left(\phi_{2}\right), R\left( \pm \phi_{2}\right)\right) \quad .
$$

The corresponding symplectic form is $d \phi_{1} \wedge d \phi_{2}$, and it is nondegenerate. Since $\operatorname{rank} \hat{A}_{0}=2$, there will be 4 fermionic moduli. Also in this sector one can use the exponential map to construct the moduli with canonical PB.

\section{Conclusions}

The structure of the total moduli space is essentially determined by the bosonic moduli associated with the maximal bosonic subgroup. This is reminiscent of the fact that the supergroups have no extra topology associated to the fermionic sector [18]. Note that the bosonic moduli space can have quite a complicated topology, (e.g. non-Hausdorff, see [8]). It would be interesting to see how the bosonic topology affects the fermionic moduli topology.

The fermionic moduli appear only in special sectors of the bosonic moduli space. In the $O S p(m \mid 2 n)$ case this condition is given by the vanishing of 
a determinant coming from the c-number part of the holonomies associated to Abelian subgroups of the maximal bosonic subgroup. In the exponential sector this condition becomes a vanishing determinant of the Abelian subalgebra generator in the fermion-fermion block of the super-adjoint representations. This is true in the general case for the exponential sector. One can use the homogeneous connections to describe the exponential sector, and the example given in the section 7 for a non-exponential holonomy shows how the constant connections could be used to describe the non-exponential sector by the moduli which have canonical PB.

The fermionic canonical moduli $\psi$ are not gauge invariant, and one can obtain gauge invariant fermionic moduli $\tilde{\psi}$ via canonical transformation. The non-exponential sector can be examined through the algebra of holonomies, and one can use the results for the $O S p$ case to study the algebra. It is not clear how the supertraces in [11] capture the properties of the fermionic moduli, since the relevant holonomies have supertraces which are c-numbers, and not elements of $B_{N}^{+}$. To understand this point it would be helpful to study the algebra of the holonomy matrices (83).

Another interesting question is how to represent $U$ in terms of basic canonical variables. This can be worked out in the exponential sector, and in order to generalise these results to the non-exponential sector and higher genus surfaces one would need a procedure for reconstructing the connection from the holonomies in the case of non-compact groups and higher-genus surfaces. Note that such a construction exists for compact and semisimple groups and surfaces of arbitrary genus [19]. Also in principle the explicit correspondence between holonomies and general connections described in [20] could be adapted to the situation at hand.

\section{Acknowledgements}

A. M. was supported by the grant PRAXIS/BCC/18981/98 from the Portuguese Foundation for Science and Technology (FCT). R. P. was supported by Financiamento Plurianual provided by the FCT. This work was supported by the programme POCTI of the FCT, co-financed by the European 
Community fund FEDER.

\section{References}

[1] S. Carlip, Quantum gravity in $(2+1)$ dimensions, Cambridge University Press, Cambridge (1998)

[2] E. Witten, Nucl. Phys. B 311 (1988/89) 46

[3] A. Achucarro and P.K. Townsend, Phys. Lett. B 180 (1986) 89

[4] M. Gunaydin, G. Sierra and P.K. Townsend, Nucl. Phys. B 264 (1986) 429

[5] K. Koehler, F. Mansouri, C. Vaz and L. Witten, Mod. Phys. Lett. A 5 (1990) 935

[6] M. Henneaux, L. Maoz and A. Schwimmer, Annals Phys. 282 (2000) 31

[7] M. Atiyah, The geometry and physics of knots, Cambridge University Press, Cambridge (1990)

[8] D. Marolf and J. Louko, Class. Quant. Grav. 11 (1994) 311

[9] B. de Wit, H.-J. Matschull and H. Nicolai, Phys. Lett. B 318 (1993) 115

[10] J.E. Nelson and T. Regge, Nucl. Phys. B 328 (1989) 190, J.E. Nelson, T. Regge and F. Zertuche, Nucl. Phys. B 339 (1990) 516

[11] L.F. Urrutia, H. Waelbroeck and F. Zertuche, Mod. Phys. Lett. A 29 (1992) 2715

[12] J.E. Nelson and R.F. Picken, Phys. Lett. B471 (200) 367

[13] F.A. Berezin, Introduction to algebra and analysis with anticommuting variables, Moscow University Press (1983)

[14] M. Atiyah and R. Bott, Phil. Trans. Roy. Soc. London Ser. A, 308 (1983) 523 
[15] L. Faddeev, Theor. Math. Phys. 1 (1969) 1

[16] I.A. Batalin and E.S. Fradkin, Phys. Lett. B 122 (1983) 157

[17] M. Henneaux, Phys. Rept. 126 (1985) 1

[18] R. Picken, J. Phys. A: Math.Gen. 16 (1983) 3457

[19] C. King and A. Sengupta, J. Math. Phys. 35 (1995) 5338

[20] M. Mackaay and R. Picken, The holonomy of gerbes with connections, preprint math.DG/0007053, to appear in Adv. Math. 